Revue internationale P.M.E.

Économie et gestion de la petite et moyenne entreprise

\title{
La présence d'un conseil d'administration ou d'un " quasi-CA » dans la PME manufacturière québécoise
}

\section{Josée St-Pierre et Luc Robitaille}

Volume 8, numéro 1, 1995

URI : https://id.erudit.org/iderudit/1008275ar

DOI : https://doi.org/10.7202/1008275ar

Aller au sommaire du numéro

Éditeur(s)

Presses de l'Université du Québec

ISSN

0776-5436 (imprimé)

1918-9699 (numérique)

Découvrir la revue

Citer cet article

St-Pierre, J. \& Robitaille, L. (1995). La présence d'un conseil d'administration ou d'un « quasi-CA » dans la PME manufacturière québécoise. Revue internationale P.M.E., 8(1), 57-80. https://doi.org/10.7202/1008275ar
Résumé de l'article

Cette étude descriptive démontre l'existence de conseil d'administration dans les PME manufacturières québécoises. Un sondage téléphonique effectué auprès de 362 PME manufacturières québécoises révèle que le CA est utilisé dans $29 \%$ de celles-ci comparativement à $14 \%$ pour les CG. Toutefois, $57 \%$ des PME interrogées affirment n'utiliser ni l'un ni l'autre à cause principalement de leur petite taille. La taille est un facteur déterminant de la présence d'un CA alors que c'est le secteur industriel qui détermine si l'on va ou non utiliser un CA ou un CG. Finalement, on constate une corrélation significative entre l'âge de l'entreprise et l'utilisation d'un comité (CA ou CG).
Tous droits réservés @ Presses de l’Université du Québec, 1995
Ce document est protégé par la loi sur le droit d'auteur. L'utilisation des services d’Érudit (y compris la reproduction) est assujettie à sa politique d'utilisation que vous pouvez consulter en ligne. 


\title{
La présence d'un conseil d'administration ou d'un « quasi-CA » dans la PME manufacturière québécoise ${ }^{1}$
}

\author{
Josée ST-PIERRE* \\ LUC ROBITAILLE* \\ Université du Québec à Trois-Rivières
}

MOTS CLÉS

Conseil d'administration - PME - Régie d'entreprises - Quasi-CA

\begin{abstract}
RÉSUMÉ
Cette étude descriptive démontre l'existence de conseil d'administration dans les PME manufacturières québécoises. Un sondage téléphonique effectué auprès de 362 PME manufacturières québécoises révèle que le CA est utilisé dans $29 \%$ de celles-ci comparativement à $14 \%$ pour les CG. Toutefois, $57 \%$ des PME interrogées affirment n'utiliser ni l'un ni l'autre à cause principalement de leur petite taille. La taille est un facteur déterminant de la présence d'un CA alors que c'est le secteur industriel qui détermine si l'on va ou non utiliser un $C A$ ou un CG. Finalement, on constate une corrélation significative entre l'âge de l'entreprise et l'utilisation d'un comité (CA ou CG).
\end{abstract}

* Josée St-Pierre est titulaire d'un doctorat en finance (Ph.D.) de l'Université Laval. Elle est l'auteure de plusieurs articles scientifiques dans les domaines de la régie d'entreprises, des offres publiques d'achat et du financement par projet. Ses intérêts de recherche portent également sur l'étude des problèmes financiers des PME. Elle est membre du GREPME depuis 1993.

** Luc Robitaille est détenteur d'un doctorat de l'Université de Montpellier I. Ses intérêts de recherche portent principalement sur la prise de décision individuelle et sur la gestion de la PME. Il a également œuvré plusieurs années comme cadre bancaire. Il est membre du GREPME depuis 1992.

Adresse : Département des sciences de la gestion et de l'économie, Université du Québec à Trois-Rivières, C.P. 500, Trois-Rivières (Québec) G9A 5H7.

1. Les auteurs aimeraient remercier le Fonds institutionnel de recherche de l'Université du Québec à Trois-Rivières, le CRSH, le FCAR ainsi que le Conseil canadien de la petite entreprise qui ont participé au financement de cette recherche. Les auteurs remercient également les professeurs P. A. Julien et Louis Raymond pour leurs commentaires pertinents sur les versions antérieures de ce texte, ainsi que les participants au séminaire de recherche « Joseph Chicha » de l'UQTR. Les auteurs demeurent les seuls responsables de toute erreur présente dans le document. 


\begin{abstract}
This descriptive research shows the extend to which boards of directors are implement in Quebec's small businesses. A poll performed through 362 small manufacturing businesses shows that $29 \%$ of them has a board of directors whereas $14 \%$ has a "quasi-board". However, $57 \%$ of small businesses interviewed uses none of them pretexting of their small size. The size of the company is a significant variable as to whether or not they would have a board while the industry they are in will influence the decision to have a board over a "quasi-board". Finally, there is a significant correlation between the age of the company and the fact to have or not a board or a "quasi-board".
\end{abstract}

\title{
RESUMEN
}

Este estudio descriptivo muestra el grado de utilización del consejo de administración en las PME quebequences. Un sondage telefónico efectuado con 362 PME manufactureras quebequences revela que el CA es utilizado en $29 \%$ comparativamente a un $14 \%$ para los CG ; mientras que $57 \%$ de los PME interrogados afirman no utilizan ninguno de los dos debido principalmente a su pequeño tamaño. El tamaño es un factor determinante del grado de utilizacion de un CA. El sector industrial indica si va o no a utilizar un $C A$ o un CG. Finalmente se constata una correlacion positiva entre la edad de la empresa y la utilizacion de un comité (CA ou CG).

\section{Introduction}

Les petites et moyennes entreprises (PME) occupent une place importante dans notre économie. Leurs modes de fonctionnement et les problèmes auxquels elles font face diffèrent de ceux des grandes entreprises. Notamment, on affirme que leur taille les empêche d'avoir recours aux services de personnel hautement qualifié pour les aider dans certaines de leurs activités. Mais devant la croissance de la complexité de leur environnement et devant les exigences en matière de compétitivité qu'entraîne la globalisation des marchés, le besoin pour les PME de se procurer des ressources humaines bien adaptées à leur nouvelle réalité se fait croissant.

Ne disposant pas des atouts nécessaires pour se procurer facilement et à faible coût des ressources humaines qualifiées, l'utilisation d'un conseil d'administration $(\mathrm{CA})^{2}$ peut constituer une solution peu coûteuse et efficace pour

2. Dans ce texte, le conseil d'administration est défini comme étant un groupe de personnes formé légalement d'après les statuts et règlements d'une entreprise constituée en société par actions (compagnie), qui se réunit entre 2 et 24 fois par année. Il est considéré que le CA se réunissant plus souvent joue plutôt le rôle d'un comité exécutif alors que celui qui ne le fait qu'une seule fois assume une obligation de présence légale sans jouer de rôle significatif dans l'organisation. Les rôles et la composition sont décrits dans les sous-sections 1.1 et 1.2 . 
les PME (Jain, 1980 ; Castaldi et Wortman, 1984 ; Richard, 1989 ; Gasse et Carrier, 1992). Cependant, ces firmes écartent souvent l'utilisation du CA parce qu'elles en sous-évaluent l'importance ou craignent une perte d'indépendance. Par ailleurs, certains auteurs mentionnent que le $\mathrm{CA}$ formel répond mal aux besoins et aux réalités de la PME. On lui substituerait plutôt un «quasi$\mathrm{CA} \gg$ ou comité de gestion $(\mathrm{CG})^{3}$ qui a moins d'autorité formelle et qui permet également de contourner les problèmes de responsabilité légale des administrateurs à l'égard des décisions prises dans l'entreprise (Fox, 1984 ; Robitaille et St-Pierre, 1993). Finalement, les entreprises qui ont accepté de créer un CA ou un CG limiteraient la venue d'administrateurs externes parce qu'elles ne les jugent pas suffisamment intéressés à l'organisation pour formuler des recommandations intéressantes et «crédibles » (Roseinstein, 1988). Cela est tout à fait particulier aux PME, puisque les grandes entreprises ont recours majoritairement à de tels administrateurs (voir entre autres Shivdasani, 1993 ; SpencerStuart, 1993 ; St-Pierre et al., 1994).

Malgré tous les avantages que l'on confère à la création d'un CA bien structuré, dont le rôle n'est pas simplement accessoire, on reconnaît que cette ressource est encore mal connue et, par conséquent, mal utilisée par les PME. C'est donc cet aspect de la gestion des PME que nous avons voulu étudier par une enquête sur sa présence dans les entreprises de petite et moyenne dimensions. Nous croyons que des variables telles que la taille de l'entreprise, sa date de création ainsi que la complexité du secteur industriel dans lequel elle évolue peuvent jouer un rôle significatif sur la décision de créer un CA ou même un CG.

Avant de soumettre les résultats de cette recherche sur l'utilisation du CA dans les PME, nous allons présenter succinctement les connaissances théoriques et empiriques que nous offrent les recherches antérieures sur certaines dimensions des conseils d'administration. Dans la première section, nous allons nous intéresser à deux aspects du conseil d'administration : les rôles dévolus au CA et sa composition. Nous supposons que ces deux aspects ne sont pas totalement indépendants puisque la composition du CA sera directement liée aux rôles qu'on souhaite qu'il assume. La deuxième section sera consacrée à la description de la méthodologie et la troisième, à l'analyse des résultats empiriques. Finalement, certains commentaires seront formulés dans la dernière section et des avenues de recherche seront proposées.

3. Dans ce texte, le comité de gestion est défini de la même façon que le CA, à l'exception qu'il peut œuvrer à l'intérieur d'une entreprise constituée ou non en société par actions et qu'aucune obligation légale n'en dicte la création. 


\section{Un conseil d'administration adapté à la PME}

Les différentes études qui traitent des CA le font surtout en contexte de grande entreprise (GE) et les recherches empiriques qui se sont intéressées au CA de la PME sont très rares considérant, entre autres, le côté plutôt confidentiel de celui-ci. Mentionnons qu'il existe plusieurs contradictions dans les conclusions des ouvrages consultés. Ce manque d'unanimité s'explique par la complexité des phénomènes entourant le $\mathrm{CA}$, par le caractère confidentiel qui nuit à son étude, par les approches théoriques et méthodologiques différentes utilisées par les chercheurs et, finalement, par la grande diversité des contextes dans lesquels il exerce son rôle.

\subsection{Les rôles attendus du conseil d'administration dans la PME}

Dans la grande entreprise, les rôles du CA peuvent être regroupés sous sept rubriques distinctes (Mace, 1986) :

1) protéger les intérêts des actionnaires ;

2) participer à la formulation de la stratégie ;

3) participer aux décisions importantes ;

4) assurer l'interface avec l'environnement ;

5) évaluer le travail de la direction ;

6) assurer la pérennité de l'entreprise ;

7) conseiller la direction.

Dans la PME, certains de ces rôles seraient plutôt illusoires étant donné que le propriétaire dirigeant est souvent en même temps le principal actionnaire. Ainsi, le rôle de protection des intérêts des actionnaires n'y serait plus nécessaire alors que celui relié à l'évaluation du travail de la direction n'y serait pas joué en considération du statut particulier du propriétaire-dirigeant.

D'après son expérience et ses observations à titre de consultant, Richard (1989) affirme que le principal rôle joué par le CA dans la PME est celui de conseiller la direction sur des problèmes concernant davantage les affaires courantes que les décisions stratégiques de l'entreprise. D'autres auteurs considèrent que le CA devrait être plus actif et jouer un rôle plus important afin de justifier pleinement sa création dans la PME. Jain (1980) confirme que le CA devrait aider à la formulation des politiques de la compagnie et conseiller la direction sur de nouvelles perspectives, orientations et sur de nouveaux objectifs. Pour ce faire, le CA devrait passer d'un rôle illusoire passif à un rôle 
plutôt actif. Roseinstein et al. (1993) affirment, à partir d'un groupe de PME financées par du capital de risque, que les administrateurs externes des CA de PME de haute technologie sont d'une grande utilité d'abord pour conseiller l'équipe de direction et ensuite pour assurer l'interface avec l'environnement financier. Les autres rôles sont plutôt marginaux. On pourrait donc s'attendre à ce qu'un CA actif adapté à la PME assume les rôles suivants :

1) participer à la formulation de la stratégie ;

2) assurer l'interface avec l'environnement ;

3) conseiller la direction.

\subsubsection{Participer à la formulation de la stratégie et aux décisions importantes}

Ce rôle consiste à fixer les objectifs, participer aux plans stratégiques, autoriser les décisions stratégiques et déterminer les politiques générales (Jain, 1980). Certains auteurs considèrent que le CA de la PME devrait jouer un rôle plus actif que celui joué symboliquement, et selon lequel il est perçu comme un organe d'approbation (Champoux-Paillé, 1992). Les différents auteurs s'entendent quant à la présence de ce rôle ; toutefois, leurs positions divergent quant à l'intensité de la participation du CA aux décisions importantes, allant d'une simple participation symbolique à un rôle plus actif (Baysinger et Hoskisson, 1990). La contribution du CA aux décisions importantes peut avoir différentes formes et intensités, selon le pouvoir que l'on veut bien lui accorder. Dans une entreprise dirigée par le principal actionnaire, comme c'est le cas de la plupart des PME, on doit s'attendre à ce que le rôle du CA se limite à celui de conseiller, au mieux à toutes les étapes du processus décisionnel, au pire après que la décision a été prise. Dans les entreprises financées par du capital de risque, Roseinstein et al. (1993) ont mis en évidence le rôle très actif du CA composé principalement de membres externes, représentants des investisseurs.

\subsubsection{Assurer l'interface avec l'environnement}

Pendant que l'environnement de la PME devient de plus en plus complexe, sa capacité à obtenir des conseils éclairés de la part d'un réseau d'experts externes s'inscrit de plus en plus comme un facteur de succès déterminant (Gasse et Carrier, 1992 ; Julien et Raymond, 1991 ; Smeltzer et al., 1991). Que ce soit pour acquérir plus de crédibilité et inspirer plus de confiance à ses partenaires, pour améliorer son réseau ou pour obtenir un meilleur éclairage sur son environnement, ce rôle particulier nous paraît tout à fait pertinent en contexte de PME. 


\subsubsection{Conseiller la direction}

Afin d'assurer la pérennité de l'entreprise, ce rôle est surtout assumé par les administrateurs externes qui agissent comme conseillers (Robinson, 1982 ; Roseinstein et al., 1993 ; Roseinstein, 1988). Les impacts de ce rôle seront d'autant plus grands que le CA aura du pouvoir (Pearce II et Shaker, 1991) et que le propriétaire-dirigeant saura démontrer une ouverture d'esprit. Les besoins de conseils externes chez la PME se font sentir tant sur le plan opérationnel que stratégique. Les besoins de conseil sur les opérations se retrouveraient surtout chez les petites entreprises, alors que les moyennes auraient besoin d'expertise plutôt sur les aspects stratégiques. Le CA peut être amené à jouer ce rôle. Il peut s'agir de guider la direction dans le choix de ses objectifs ou sur des questions concernant l'économie, la concurrence, la technologie, la législation ou la gestion. Le CA sera donc constitué de différents membres pouvant fournir à l'entreprise une expertise dans des domaines où elle ne peut employer du personnel compétent à temps plein.

D'une façon générale et selon la taille de l'entreprise, les principaux rôles joués par le CA pourraient se résumer à ceux de soutenir le propriétaire-dirigeant dans ses activités ainsi que dans sa solitude, et de le conseiller dans sa gestion, les stratégies ainsi que dans les opérations courantes de son entreprise.

\subsection{La composition du conseil d'administration}

Cette dimension du CA a fait l'objet de nombreuses études. La disponibilité des données n'est d'ailleurs pas étrangère à cette constatation. Les typologies reposant sur la nature des membres du CA se limitent à distinguer les membres internes des membres externes et ceux qui possèdent ou non des actions. Shivdasani (1993) a redéfini les classes d'administrateurs externes en identifiant ceux qui avaient des liens de parenté ou d'affaires avec l'entreprise et les a alors classés comme des administrateurs externes-associés représentant le point de vue des dirigeants plutôt que celui des actionnaires. À cette définition élargie, St-Pierre et al. (1994) ont ajouté un quatrième groupe représentant bien l'économie canadienne où il existe de nombreux liens de parenté entre les grandes entreprises. Ils ont donc défini des «administrateurs externesgroupe », groupe dans lequel on retrouve les administrateurs d'une compagnie qui siègent également à d'autres CA de compagnies faisant partie d'un même empire, c'est-à-dire étant sous le contrôle direct ou indirect de la même maison mère.

L'impact des administrateurs externes sur l'efficacité des CA est équivoque. Certains avancent qu'il est très positif alors que d'autres soutiennent le contraire. Ceux qui prétendent que la présence d'administrateurs externes 
est bénéfique à l'entreprise font reposer leur argumentation sur les éléments suivants : leur présence permet une meilleure surveillance de la direction, procure une plus grande connaissance de l'environnement et offre une certaine assurance d'objectivité (Jain, 1980 ; Nelson, 1985 ; Brickley et James, 1987 ; Roseinstein et Wyatt, 1990).

Jensen (1994) suggère que le seul administrateur interne devant participer activement au CA est le président de la compagnie ; les autres membres de la compagnie ne devraient intervenir que lors de discussions particulières.

Dans leur étude sur des PME de haute technologie, les PDG des sociétés interrogées par Roseinstein et al. (1993) ont indiqué que les administrateurs externes (provenant surtout de leur investisseur/créancier) étaient particulièrement utiles pour surveiller la performance financière de l'entreprise, animer et aider le conseil et voir à la succession du PDG. Par contre, certains notent que les entreprises privilégiant une domination des administrateurs internes présentent de meilleures performances. S.J. Ford (1980) remarque que les internes sont de plus en plus prêts à confronter le président du conseil. R.H. Ford (1987, 1988) observe d'ailleurs que les CA composés uniquement d'administrateurs internes ont plus d'influence sur la gestion de l'entreprise que les CA mixtes. Cette observation peut s'expliquer par la meilleure connaissance que les internes ont des activités quotidiennes de l'entreprise, ce qui leur permet de mieux juger de la stratégie (Baysinger et al., 1991). En résumé, le débat sur la composition idéale du CA autant chez la grande entreprise que chez la PME est loin d'être clos.

Alors que dans la GE les administrateurs externes occupent généralement les fonctions de cadres supérieurs dans d'autres GE (Korn/Ferry, 1990 ; Shivdasani, 1993 ; St-Pierre et al., 1994), ceux de la PME devraient surtout être des propriétaires-dirigeants (Groupement québécois d'entreprises, 1987). Ils devraient posséder des qualités complémentaires à celles du dirigeant et avoir des contacts et des expériences aptes à combler les lacunes de la PME (Jain, 1980 ; Danco et Jonovic, 1981). Castaldi et Wortman (1984) considèrent que pour jouer correctement les rôles décrits plus haut et entre autres pallier les lacunes de la PME en matière de ressources humaines, les administrateurs des CA devraient représenter les actionnaires, la communauté des affaires et l'environnement légal, financier et technologique de l'entreprise. Ce conseil d'administration type composé de sept membres ${ }^{4}$ peut être dominé ou

4. Cette taille nous semble relativement élevée pour une PME. Même dans la grande entreprise, on reconnaît l'inefficacité des CA comptant un trop grand nombre d'administrateurs. D'ailleurs, à cet effet, SpencerStuart (1993) ont observé entre 1983, 1988 et 1993 une diminution notable de la taille des CA passant de 16 à 15 à 14 membres respectivement et une croissance de l'importance relative des administrateurs externes. On préfère un conseil de plus petite taille et plus actif qu'auparavant. 
non par des employés de l'entreprise. On devrait au minimum retrouver un gestionnaire externe, un expert financier externe ainsi qu'un expert économique pouvant venir du milieu académique. Cette composition assure un juste équilibre interne/externe tout en permettant à l'entreprise de combler ses lacunes en matière de ressources humaines. Les différents types d'expertise recherchés servent à renforcer les faiblesses reconnues historiquement chez les PME.

En plus de la composition optimale du CA, Castaldi et Wortman (1984) suggèrent l'utilisation de différents comités. Ceux-ci procurent une meilleure allocation des ressources étant donné l'appariement entre les compétences de chacun des administrateurs et les besoins particuliers de l'entreprise. Ces comités existent dans la plupart des grandes entreprises, mais aucune étude n'a encore démontré leur utilisation chez les PME. Jain (1980) confirme l'importance de renforcer le CA en y faisant participer des administrateurs externes ayant également cette expertise complémentaire recherchée. Il recommande de recruter de tels administrateurs chez les professionnels, les chercheurs scientifiques, les universitaires, les hauts dirigeants d'importantes sociétés oeuvrant dans un environnement technologiquement complexe, les consultants, les administrateurs institutionnels ainsi que chez d'anciens dirigeants retraités.

\subsection{Une alternative adaptée au contexte de la PME : le "quasi-CA "}

Certains auteurs affirment que le CA est trop formel pour répondre correctement aux besoins et réalités de la PME. Fox (1984) suggère plutôt la création de comités consultatifs ou de gestion, soit des « quasi-CA », qui permettrait, entre autres, de contourner les problèmes de responsabilité légale des administrateurs. Cette responsabilité oblige l'entreprise à se doter d'un contrat d'assurance dont les coûts peuvent sembler élevés pour une PME. Ces comités permettraient également d'attirer plus de conseillers externes à l'entreprise. Mais ce qui semble plaire davantage aux dirigeants de PME, c'est l'absence d'autorité formelle de tel comité à l'égard de l'entreprise. Le "quasi-CA » conseille et ne gouverne pas l'entreprise.

La recension qui précède a permis de décrire comment le CA ou le CG dans le cas des PME devrait être composé. Toutefois, on ignore jusqu'à quel point cette composition convient à ces entreprises puisqu'aucune étude empirique n'a permis, jusqu'à maintenant, de vérifier son existence. C'est donc cette problématique de recherche que nous avons voulu examiner dans cette étude descriptive qui ne prétend pas faire toute la lumière sur ce phénomène complexe, mais vise plutôt à vérifier si les entreprises utilisent cet outil de 
gestion qu'est le CA et qui peut s'avérer intéressant dans l'environnement économique en mutation que connaissent les PME depuis plusieurs années.

\section{L'existence d'un CA adapté à la PME : hypothèses et échantillon}

\subsection{Hypothèses}

Les études citées précédemment, ainsi que celle de Robitaille et St-Pierre (1993), permettent de formuler quelques hypothèses générales quant à l'utilisation du CA ou du CG dans la PME. L'étude exploratoire de Robitaille et St-Pierre (1993) effectuée auprès d'un échantillon non aléatoire de neuf administrateurs de PME a permis de développer une hypothèse quant à l'utilisation du CA et la taille de l'entreprise. En effet, les auteurs mentionnent que les PME de petite taille auraient tendance à privilégier l'utilisation d'un comité de gestion. On le préférerait au CA parce qu'on craint le cadre juridique de ce dernier et qu'on tient à dégager ses membres de toute responsabilité face aux agissements de l'entreprise, ce qui ne peut qu'en faciliter le recrutement. Le CA formel serait surtout utilisé par les moyennes entreprises, comptant entre 100 et 500 employés, et celles qui possèdent des actionnaires non familiaux ou institutionnels. La première tendance observée semble donc établir une corrélation positive entre la taille de l'entreprise et l'utilisation d'un CA ou d'un CG.

\subsubsection{Première hypothèse: I/ existe une relation positive entre la taille de l'entreprise et la présence d'un conseil d'administration ou d'un comité de gestion.}

Par ailleurs, on pourrait s'attendre à ce que le degré de formalisme atteint dans une entreprise selon les stades de croissance pourrait l'amener à créer un CA ou un CG permettant à l'entrepreneur d'élargir considérablement son réseau d'information et d'aide. Ne disposant pas du niveau de maturité, nous pouvons utiliser l'âge de l'entreprise comme le suggèrent Gasse et Carrier (1992) qui résument les relations entre le degré de maturité de l'entreprise et la date de sa création ${ }^{5}$. On pourrait alors faire l'hypothèse selon laquelle il y a plus de chances de retrouver des CA dans les entreprises plus âgées, aucun CA ni CG dans les entreprises plus jeunes et un CG dans les entreprises se situant entre ces deux niveaux.

5. Voir Gasse et Carrier (1992), chapitre 1. 


\subsubsection{Deuxième hypothèse : II existe une relation positive entre l'âge de l'entreprise et la présence d'un conseil d'administration ou d'un comité de gestion.}

Finalement, plusieurs auteurs ont mentionné que le besoin d'expertise spécifique devrait favoriser la création d'un conseil d'administration ou d'un comité de gestion pour les entreprises qui n'ont pas les moyens d'embaucher de telles ressources dont les PME. On peut, dès lors, supposer que la complexité du secteur industriel de l'entreprise devrait accroître ses besoins en experts et favoriser la création d'un CA ou d'un CG.

\subsubsection{Troisième hypothèse (a) : Il existe une relation positive entre la complexité du secteur industriel de la PME et la présence d'un conseil d'administration ou d'un comité de gestion.}

De plus, cette même complexité devrait amener un plus grand nombre d'administrateurs externes à faire partie de ces comités.

\subsubsection{Troisième hypothèse (b) : II existe une relation positive entre la complexité du secteur industriel de la PME et l'importance relative du nombre de membres externes composant le conseil d'administration ou le comité de gestion.}

\subsection{Constitution de l'échantillon}

Afin de vérifier les hypothèses précédentes, nous avons réalisé un sondage téléphonique auprès de 492 entreprises manufacturières québécoises comptant plus de 10 employés listées dans le Répertoire des entreprises manufacturières 1993, Région Mauricie-Bois-Francs-Drummond (Québec). Nous avons demandé à discuter avec le propriétaire-dirigeant ou, en son absence, avec une personne assumant des responsabilités de direction. Sur les 492 téléphones effectués, 113 dirigeants de compagnie n'ont pu être rejoints après plusieurs appels et seulement $4 \%$ des personnes rejointes ont refusé de nous accorder quelques minutes pour répondre à un court questionnaire. Le questionnaire visait à constituer une liste d'entreprises qui accepteraient de participer à une enquête beaucoup plus exhaustive relativement à l'utilisation du CA dans la PME. Étant donné les objectifs du sondage téléphonique, nous n'avons posé que quelques questions fermées permettant de caractériser le type d'entreprises utilisant ou non un CA. 
L'appel téléphonique durait environ 10 minutes selon que l'entreprise affirmait utiliser un CA, un CG ou ni l'un ni l'autre. Les statistiques recueillies sont donc relativement peu détaillées mais permettent tout de même de dégager une image sommaire de l'entreprise type qui utilise les services d'un conseil d'administration. Voici comment se répartit l'échantillon :

TABLEAU 1

Constitution de l'échantillon

\begin{tabular}{lc}
\hline Entreprises non rejointes après 3 ou 4 appels & 113 \\
Entreprises ayant refusé de nous accorder quelques minutes au téléphone & 17 \\
\hline Entreprises utilisant un CA & 105 \\
Entreprises utilisant un CG & 49 \\
Entreprises n'utilisant ni CA ni CG & $208^{*}$ \\
Entreprises constituant l'échantillon & 362 \\
\hline
\end{tabular}

Source : Répertoire des entreprises manufacturières 1993, Région Mauricie-Bois-FrancsDrummond.

* Plusieurs entreprises ayant affirmé utiliser un CA ou un CG ont été reclassées étant donné la fréquence annuelle des réunions de leur comité (nous en rediscuterons plus loin).

Contrairement à nos attentes, un nombre plus important d'entreprises de petite et de moyenne taille utilise un CA plutôt qu'un comité alternatif (CG), soit $29 \%$ contre $14 \%$. Les entreprises qui n'utilisent ni les services d'un CA ni ceux d'un CG totalisent $57 \%$ des répondants. Il est intéressant de connaître pourquoi la majorité des entreprises n'utilisent ni CA ni CG et de voir si cette décision peut s'expliquer par certaines variables communes : c'est ce dont nous traitons ci-après.

\section{Analyse des résultats}

\subsection{Description des trois groupes d'entreprises}

Afin de valider l'affirmation, à savoir que certaines entreprises disaient utiliser un CA ou un CG de façon formelle, nous avons demandé aux personnesressources combien de fois par année se réunissait leur comité. Certaines entreprises (27) ont affirmé ne réunir leur CA qu'une seule fois par année, ce qui nous porte à croire que ces entreprises ont créé un CA par obligation légale et qu'elles ne l'utilisent pas comme il est d'usage de le faire. Par ailleurs, d'autres entreprises ayant confirmé la présence d'un CA ou d'un CG ont indiqué se réunir au moins une fois par semaine. Cette affirmation reflète davantage les caractéristiques d'un comité exécutif. Nous avons donc classé ces entreprises comme ayant ni CA ni CG, faisant passer le groupe ayant affirmé avoir un CA de 139 à 105 entreprises et celui utilisant un CG de 60 à 49. 
Nous étudierons donc 208 entreprises n'utilisant de façon formelle ni l'un ni l'autre des comités.

\subsubsection{Les entreprises qui n'ont ni conseil d'administration, ni comité de gestion}

Nous avons pu recenser 163 entreprises qui ont affirmé ne pas utiliser les services d'un CA ni d'un CG. Celles-ci ont en moyenne 21 ans, ont un effectif moyen de 27 employés et un chiffre d'affaires se situant aux environs de 1 million de dollars. Le répondant (souvent le PDG) a entre 41 et 50 ans et détient un diplôme d'études secondaires. L'ensemble de ces entreprises n'a jamais eu de CA ni de CG et ne prévoit pas en créer un à court terme. La majorité des répondants considèrent qu'un tel comité leur serait inutile et ils affirment ne pas en avoir besoin. Plusieurs raisons qui auraient pu justifier la décision de ne pas utiliser un CA ou un $\mathrm{CG}$ ont été proposées aux répondants ${ }^{6}$. De celles-ci, seule la taille de leur entreprise a été retenue comme facteur explicatif.

\subsubsection{Les entreprises ayant un conseil d'administration (CA)}

Ces entreprises ont en moyenne 26 ans et emploient 45 personnes. Elles recourent aux services de leur CA depuis environ 11 ans, et celui-ci est composé de 4 personnes dont 3 membres internes et un externe qui se réunissent six fois par année.

À la question: Pourquoi n'avez-vous pas créé un comité de gestion plutôt qu'un CA formel ? les dirigeants ont majoritairement répondu préférer un CA parce qu'ils voulaient qu'il ait plus de pouvoir et d'autorité. De plus, la responsabilité légale de leur $\mathrm{CA}$ ne les inquiète pas, contrairement aux arguments soulevés dans les recherches antérieures.

\subsubsection{Les entreprises ayant un comité de gestion (CG)}

Dans $14 \%$ des cas, les entreprises interrogées ont affirmé préférer un comité de gestion au conseil d'administration. Ces entreprises ont en moyenne 29 ans, comptent 32 employés et utilisent un CG depuis environ 9 ans. Celui-ci est composé de 5 personnes qui sont presque toutes des employés de l'entreprise, et il se réunit en moyenne dix fois par année.

6. Les raisons suggérées sont : 1) leur méconnaissance de tels comités, 2) le temps que cela nécessite, 3) le coût trop élevé de fonctionnement, 4) leur souci de confidentialité, 5) la possibilité de se faire imposer des décisions et 6) la taille de l'entreprise. 
À la question: Pourquoi n'avez-vous pas créé un CA formel ? ces entreprises ont répondu que leur taille n'en justifiait pas la création. Par ailleurs, contrairement à nos attentes, les motifs invoqués précédemment tels que la responsabilité légale, l'autorité et le paiement de primes pour une assurance responsabilité ne semblaient pas avoir influencé leur décision.

Finalement, lorsque nous les avons questionnées sur leur planification à court terme, la majorité de ces entreprises ont affirmé ne pas prévoir remplacer leur comité de gestion par un CA formel.

Un tableau synoptique des quelques caractéristiques communes aux trois groupes précédents nous amène à constater qu'il semble y avoir quelques différences entre ces derniers. Nous avons repris, dans le tableau 2, les informations concernant leurs taille, année de création et secteur industriel.

Les entreprises n'utilisant ni CA ni CG semblent se distinguer des autres sur les variables de taille et d'âge, alors que la taille semble influencer également le type de comité qui sera mis sur pied.

\section{TABleau 2}

Caractéristiques de taille, d'année de création et de secteur industriel

\begin{tabular}{lccc}
\hline Entreprises ayant : & $\begin{array}{c}\text { Nombre } \\
\text { d'employés }\end{array}$ & $\begin{array}{c}\text { Année de } \\
\text { création }\end{array}$ & $\begin{array}{c}\text { Secteur } \\
\text { industriel }\end{array}$ \\
\hline Ni CA ni CG $(\mathrm{N}=208)$ & 27 & 1973 & 1,87 \\
Un comité de gestion $(\mathrm{N}=49)$ & 32 & 1966 & 2,22 \\
Un conseil d'administration $(\mathrm{N}=105)$ & 45 & 1968 & 2,10 \\
\hline
\end{tabular}

\subsection{Taille, secteur industriel, âge de l'entreprise et utilisation d'un comité}

La complexité du secteur industriel dans lequel œuvre une entreprise devrait justifier l'utilisation d'un CA (H3a) composé de plusieurs administrateurs externes (H3b), pouvant fournir une expertise complémentaire aux compétences des dirigeants. De plus, on s'attend à ce que la taille $(\mathrm{H} 1)$ et l'âge $(\mathrm{H} 2)$ de l'entreprise jouent un rôle significatif sur l'utilisation ou non d'un comité.

7. La typologie utilisée visait à regrouper les entreprises selon le degré de complexité présumé de leurs activités allant du moins complexe (secteur traditionnel $=1$ ) au plus complexe (secteur moderne $=3$ ). Une description des activités des entreprises nous a amené à cette division, qui d'ailleurs pourrait être refaite selon d'autres facteurs de complexité. Voir la liste des industries composant ces trois secteurs à l'annexe 1 . 
Pour la vérification des hypothèses $\mathrm{H} 1$ et $\mathrm{H} 2$, nous avons utilisé un modèle de régression logit qui permet de vérifier l'existence d'une relation significative entre une variable indépendante continue et une variable dépendante discrète (dichotomique). La variable dépendante peut alors être interprétée comme une probabilité dont les valeurs prédites se situent à l'intérieur de l'intervalle $[0,1]$. Nous avons réalisé deux régressions pour chaque hypothèse qui ont permis de vérifier s'il existe une différence de taille ou d'âge entre les entreprises n'utilisant ni CA ni CG et celles qui utilisent l'un ou l'autre de ces comités; et entre les entreprises qui utilisent un CG et celles qui utilisent un $\mathrm{C}^{8}$. Finalement, un test de chi carré a été utilisé pour vérifier l'hypothèse H3a. Les résultats sont présentés dans les tableaux 3, 4 et 5 .

Comme le laissait entrevoir notre première hypothèse, la taille influence significativement le choix d'utiliser ou non un comité et, à un degré moindre, le type de comité. Les entreprises n'utilisant ni un CA ni un CG sont les plus petites, celles qui utilisent un CA sont les plus grandes et celles qui font appel au $\mathrm{CG}$ sont à mi-chemin entre les deux.

Par ailleurs, on constate une relation significative entre l'année de création de l'entreprise et l'utilisation d'un comité. Ainsi que nous l'avancions dans notre deuxième hypothèse, les plus jeunes entreprises n'ont ni CA ni CG alors que celles qui utilisent ces comités sont nettement plus âgées. Cela peut s'expliquer par le fait que les jeunes entreprises n'ont pas acquis cette maturité dans leur développement qui les obligerait à modifier leur mode de fonctionnement et à adopter un mode de gestion plus formalisé 9 . Enfin, l'âge de l'entreprise ne semble pas lié au type de comité (CA ou CG) utilisé.

Le secteur industriel influence considérablement le choix d'un comité. Contrairement à l'hypothèse $\mathrm{H} 3 \mathrm{a}$, les entreprises du secteur moderne utilisent indifféremment le CA ou le CG ou même ni l'un ni l'autre. Les entreprises du premier groupe (ni CA ni CG) œuvrent majoritairement dans un secteur traditionnel où l'environnement plutôt stable ne requiert pas nécessairement la mise sur pied d'un comité quelconque pouvant conseiller la direction.

Les différences obtenues à partir de tests univariés sont confirmées sur le plan multivarié où, à partir d'un modèle de régression logit, on constate

8. Le lecteur intéressé aux détails concernant l'utilisation du modèle logit pourra consulter les travaux de Aldrich et Nelson (1986).

9. Un test de chi carré a démontré qu'il n'existe aucune relation significative entre la taille de l'entreprise et le secteur industriel et entre l'âge de l'entreprise et le secteur industriel. Par contre, les entreprises âgées de moins de 30 ans comptent significativement moins d'employés que les autres (coefficient chi carré significatif à $3.8 \%$ ). 


\section{TABleau 3}

Relation entre la présence d'un comité, le nombre d'employés et l'âge (niveau de signification de la variable)

\begin{tabular}{|c|c|c|c|c|}
\hline & \multicolumn{3}{|c|}{ Variable } & \multirow{2}{*}{$\begin{array}{c}\text { Pseudo-R }^{2} \\
\text { ou RV* }\end{array}$} \\
\hline & Constante & $\begin{array}{c}\text { Nombre } \\
\text { d'employés }\end{array}$ & $\begin{array}{c}\text { Année de } \\
\text { création }\end{array}$ & \\
\hline $\begin{array}{l}\mathrm{Ni} \mathrm{CA} / \mathrm{CG} \text { ou } \mathrm{CA} / \mathrm{CG}^{1} \\
(\mathrm{~N}=362)\end{array}$ & $\begin{array}{l}-0,8037 \\
(0,0000)\end{array}$ & $\begin{array}{c}0,0155 \\
(0,0003)\end{array}$ & & $\begin{array}{c}102,15 \\
(0,0112)\end{array}$ \\
\hline $\begin{array}{l}\mathrm{Ni} C A / C G \text { ou } \mathrm{CA} / \mathrm{CG}^{1} \\
(\mathrm{~N}=272)\end{array}$ & $\begin{array}{l}27,2103 \\
(0,0394) \\
\end{array}$ & & $\begin{array}{r}-0,0142 \\
(0,0342) \\
\end{array}$ & $\begin{array}{c}74,17 \\
(0,1032) \\
\end{array}$ \\
\hline $\begin{array}{l}C G \text { ou } \mathrm{CA}^{2} \\
(\mathrm{~N}=154)\end{array}$ & $\begin{array}{c}0,3272 \\
(0,2478)\end{array}$ & $\begin{array}{c}0,0117 \\
(0,0726)\end{array}$ & & $\begin{array}{l}57,21 \\
(0,4301)\end{array}$ \\
\hline $\begin{array}{l}\text { CG ou CA2 } \\
(\mathrm{N}=86)\end{array}$ & $\begin{array}{l}-8,4325 \\
(0,6665)\end{array}$ & & $\begin{array}{c}0,0047 \\
(0,6397)\end{array}$ & $\begin{array}{c}54,47 \\
(0,1320)\end{array}$ \\
\hline
\end{tabular}

* RV : Ratio de vraisemblance équivalent à la statistique $\mathrm{F}$ utilisée en régression standard. Il s'agit d'une statistique qui suit une distribution chi carré avec $\mathrm{N}-k$ degrés de liberté et qui permet de tester l'hypothèse selon laquelle tous les coefficients obtenus sont significativement différents de zéro.

1. La variable dépendante de ce modèle prend la valeur de 1 si l'entreprise utilise les services d'un CA ou d'un CG, et 0 autrement.

2. La variable dépendante de ce modèle prend la valeur de 1 si l'entreprise utilise un CA et 0 si elle utilise un CG. Les autres entreprises ont été exclues de l'échantillon.

\section{TABleau 4}

Relation entre le choix du comité et le secteur industriel

( $\%$ dans la ligne) $\{\%$ dans la colonne $\}$

\begin{tabular}{|c|c|c|c|}
\hline & $\begin{array}{l}\text { Secteur traditionnel } \\
\text { (faible complexité) }\end{array}$ & $\begin{array}{l}\text { Secteur de transfor- } \\
\text { mation des ressources }\end{array}$ & $\begin{array}{l}\text { Secteur moderne } \\
\text { (forte complexité) }\end{array}$ \\
\hline $\begin{array}{l}\text { Ni CA ni CG } \\
(\mathrm{N}=208)\end{array}$ & $\begin{array}{c}85 \\
(40,87 \%) \\
\{66,41 \%\}\end{array}$ & $\begin{array}{c}65 \\
(31,25 \%) \\
\{58,04 \%\}\end{array}$ & $\begin{array}{c}58 \\
(27,88 \%) \\
\{47,54 \%\}\end{array}$ \\
\hline $\begin{array}{l}\mathrm{CG} \\
(\mathrm{N}=49)\end{array}$ & $\begin{array}{c}10 \\
(20,41 \%) \\
\{7,81 \%\}\end{array}$ & $\begin{array}{c}18 \\
(36,73 \%) \\
\{16,07 \%\}\end{array}$ & $\begin{array}{c}21 \\
(42,86 \%) \\
\{17,21 \%\}\end{array}$ \\
\hline $\begin{array}{l}\mathrm{CA} \\
(\mathrm{N}=105)\end{array}$ & $\begin{array}{c}33 \\
(31,43 \%) \\
\{25,78 \%\}\end{array}$ & $\begin{array}{c}29 \\
(27,62 \%) \\
\{25,89 \%\}\end{array}$ & $\begin{array}{c}43 \\
(40,95 \%) \\
\{35,25 \%\}\end{array}$ \\
\hline \multicolumn{3}{|c|}{$\begin{array}{l}\text { Test de chi carré } \\
\text { Coefficient Lambda }\end{array}$} & $\begin{array}{c}11,198^{* *} \\
0,090\end{array}$ \\
\hline
\end{tabular}

$* * p<0,05$ 
effectivement (tableau 5) que la taille de l'entreprise influe sur le choix du type de comité alors que la taille et, dans une moindre mesure, le secteur influencent la décision de recourir ou non à un comité. L'année de création n'a plus d'effet marginal sur le choix de constituer un comité.

\section{Tableau 5}

Variables influençant le choix du comité

(niveau de signification de la variable)

\begin{tabular}{lccccc}
\hline & \multicolumn{2}{c}{ Variable } & \multicolumn{2}{c}{$\begin{array}{c}\text { Pseudo-R } \\
\text { ou RV* }\end{array}$} \\
\cline { 2 - 5 } & Constante & $\begin{array}{c}\text { Nombre } \\
\text { d'employés }\end{array}$ & Secteur & $\begin{array}{c}\text { Année de } \\
\text { création }\end{array}$ & \\
\hline CA ou CG & $-33,6675$ & 0,0239 & $-0,1914$ & 0,0172 & 98,75 \\
$(\mathrm{~N}=86)$ & $(0,1594)$ & $(0,0432)$ & $(0,5291)$ & $(0,1536)$ & $(0,0877)$ \\
Ni CA/CG ou & 12,9785 & 0,0195 & 0,3877 & $-0,0077$ & 304,74 \\
CA/CG2 & $(0,3611)$ & $(0,0004)$ & $(0,0190)$ & $(0,2838)$ & $(0,0143)$ \\
$(\mathrm{N}=272)$ & & & & & \\
\hline
\end{tabular}

* $\quad \mathrm{RV}=$ Ratio de vraisemblance équivalent à la statistique $\mathrm{F}$ utilisée en régression standard. Il s'agit d'une statistique qui suit une distribution chi carré avec $\mathrm{N}-k$ degrés de liberté et qui permet de tester l'hypothèse selon laquelle tous les coefficients obtenus sont significativement différents de zéro.

1. La variable dépendante de ce modèle prend la valeur de 1 si l'entreprise utilise les services d'un CA et 0 si elle fait appel au CG. Les autres entreprises ont été exclues de l'échantillon.

2. La variable dépendante de ce modèle prend la valeur de 1 si l'entreprise affirme ne pas utiliser les services ni d'un CA ni d'un CG, et 0 autrement.

Sur quelques-uns des aspects précédents, on constate que les entreprises qui utilisent un CG ressemblent davantage à celles qui utilisent un CA qu'aux autres. Nous avons donc voulu vérifier cette hypothèse en comparant entre elles les entreprises de ces deux groupes ainsi que la composition de leur comité respectif.

\subsection{Différences entre les sociétés faisant appel à un CA ou à un CG}

Comme nous l'avons constaté, les entreprises qui utilisent un CA sont significativement plus grandes que celles qui préfèrent un CG (tableau 6). Les résultats de la régression logit confirment également cette hypothèse (tableau 5). Par ailleurs, la formation du comité ne cadre pas avec le comité «idéal » de Castaldi et Wortman (1984), qui suggère un comité formé d'environ 7 personnes dont un minimum de 3 administrateurs externes. En effet, la taille des comités étudiés se situait en moyenne entre 4 et 5 administrateurs dont 
moins de $17 \%$ seulement provenaient de l'extérieur de l'entreprise. Roseinstein et al. (1993) ont observé, chez des PME américaines de haute technologie financées par des sociétés de capital de risque, des CA composés en moyenne de 3 administrateurs avant le financement externe, 4,81 administrateurs lorsque l'investisseur institutionnel a fait son apport de capitaux et 5,62 administrateurs deux ans plus tard. Ils constatent une relation positive entre la taille du CA et le degré de maturité de l'entreprise, et une faible proportion d'administrateurs internes au sein de ces compagnies (30,07\%). Les PME financées par du capital de risque voient donc leur CA dominé et contrôlé majoritairement par les représentants de leurs créanciers. Richard (1989) affirme le contraire et suggère que le CA de la petite entreprise privée serait dominé par des administrateurs internes provenant du milieu familial de l'entrepreneur et de certains professionnels qui l'entourent.

Tableau 6

Différences entre les sociétés ayant un CA et celles qui ont un CG

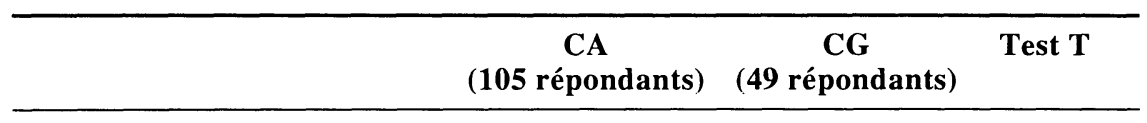

Nombre moyen d'employés dans

l'entreprise

Année de création de l'entreprise

Taille du CA/CG

$\begin{array}{ccc}45 & 32 & -1,8426^{*} \\ 1968 & 1966 & -0,4508 \\ 4,16 & 4,53 & 0,5451 \\ 16,93 & 12,43 & -1,2549 \\ 11,35 & 9,17 & -1,0236\end{array}$

Nombre d'années d'existence

du CA/CG

Nombre de réunions par année

$* \mathrm{p}<0,10 \quad * * \mathrm{p}<0,05 \quad * * * \mathrm{p}<0,01 \quad * * * * \mathrm{p}<0,001$

On retrouve un pourcentage plus important d'administrateurs externes dans le CA, mais celui-ci n'est pas significatif ${ }^{10}$. Finalement, les comités se réunissent à des fréquences totalement différentes, soit moins d'une fois par mois pour le CA et deux fois par mois pour le CG. Ces résultats diffèrent significativement de ceux de Richard (1989) qui indique une fréquence de réunion annuelle de 4 à 5 pour les sociétés privées et de 6 à 10 pour les petites entreprises en croissance. La fréquence élevée des réunions pourrait confirmer qu'on y discute probablement plus d'affaires courantes que de problèmes stratégiques. Par contre, étant donné que les deux groupes d'entreprises œuvrent

10. Lorsque les compagnies sont regroupées en fonction de leur secteur industriel, on observe que les CA comptent significativement plus de membres que les CG dans le secteur traditionnel. Toutefois, aucune autre différence intersectorielle n'apparaît dans ces études de groupes. 
dans les secteurs industriels de transformation des ressources, la complexité de l'industrie ne peut expliquer cet écart dans la fréquence des réunions. La taille et le lien qu'elle peut avoir avec le stade de développement de l'entreprise pourraient peut-être en expliquer cette différence dans le nombre de réunions par année ou, encore, les regroupements d'entreprises que nous avons effectués.

Selon l'hypothèse $3 b$ formulée précédemment, il existerait une relation entre la complexité du secteur industriel de l'entreprise et le besoin pour celleci de se doter d'un conseil d'administration ou d'un comité de gestion composé d'un nombre important d'experts externes. Nous avons donc tenté de vérifier cette hypothèse en reliant le pourcentage de membres externes dans les CA ou CG au secteur industriel. La matrice des corrélations de rang de Spearman ne fait ressortir aucune relation significative entre ces deux variables. Toutefois, la taille de l'entreprise semble, une fois de plus, influencer cette composition.

Cette matrice ne permet pas de confirmer que la complexité du secteur industriel influence la composition du comité créé ${ }^{11}$. Toutefois, le nombre de membres augmente avec la taille alors que la fréquence annuelle des réunions est plus élevée chez les sociétés faisant partie d'un secteur plus complexe ainsi que chez celles qui comptent un pourcentage plus élevé de membres externes. Ces deux derniers résultats pourraient confirmer que les compagnies qui ont besoin d'un CA, à cause de leur environnement, s'adjoignent des externes pouvant compléter l'expertise de leur personnel, et sollicitent leurs services de façon régulière.

\section{Conclusion}

Les conseils d'administration ont été l'objet de vives critiques depuis quelque temps, notamment aux États-Unis où on les a souvent comparés à des « clubs privés » où les administrateurs discutaient bien plus de leurs projets personnels que des affaires de la compagnie qui assumait le coût de leurs réunions. Les vagues de poursuite contre des administrateurs de grandes sociétés ont, par contre, ranimé les discussions quant à l'importance d'un CA efficace et dont les rôles n'étaient plus accessoires mais bien nécessaires à l'entreprise. La complexité de l'environnement, la mondialisation des marchés, la concurrence accrue et de plus en plus rapide pour envahir des marchés qui étaient jusqu'à

11. Une régression multivariée a, toutefois, démontré que la seule variable significative, parmi la taille, le secteur industriel et l'âge de l'entreprise, influençant la composition du comité était la taille. Les entreprises de plus grande taille ont tendance à avoir plus de membres externes siégeant à leur comité. Cela pouvant peutêtre confirmer les besoins de se doter d'une structure plus formelle à mesure que la taille augmente. 
Tableau 7

Matrice de corrélations de rang

\begin{tabular}{|c|c|c|c|c|c|c|}
\hline & $\begin{array}{l}\mathbf{C A}=1 \\
\mathbf{C G}=\mathbf{0}\end{array}$ & $\begin{array}{c}\text { Nombre de } \\
\text { membres }\end{array}$ & $\begin{array}{c}\% \text { de } \\
\text { membres } \\
\text { externes }\end{array}$ & $\begin{array}{c}\text { Nombre } \\
\text { de réunions } \\
\text { annuelles }\end{array}$ & $\begin{array}{l}\text { Nombre } \\
\text { d'employés }\end{array}$ & $\begin{array}{c}\text { Secteur } \\
\text { industriel }\end{array}$ \\
\hline Nombre de membres & 0,0742 & & & & & \\
\hline Pourcentage de membres externes & 0,0818 & $0,3676 * * * *$ & & & & \\
\hline Nombre de réunions par année & $-0,3408 * * * *$ & 0,0688 & $0,2520 * * *$ & & & \\
\hline Nombre d'employés & 0,1139 & $0,3208 * * * *$ & 0,0977 & $-0,0491$ & & \\
\hline Secteur industriel & $-0,0673$ & 0,0718 & 0,0977 & $0,1907 * *$ & 0,0473 & \\
\hline Année de création & 0,0820 & 0,0011 & $-0,0471$ & $-0,0606$ & $-0,3689 * * * *$ & $-0,0483$ \\
\hline
\end{tabular}

$* \mathrm{p}<0,10$

${ }^{* *} \mathrm{p}<0,05$

$* * * \mathrm{p}<0,01$

$* * * * \mathrm{p}<0,001$ 
présent tenus pour acquis obligent désormais les entreprises à réunir le plus de ressources possible pour leur permettre de survivre et de bien performer.

D'après les informations que nous avons pu recueillir, il semble que les PME soient conscientes de leur besoin et de cet impératif de combler leurs lacunes en ressources humaines, par la création d'un CA ou d'un comité qui jouerait les mêmes rôles sans en avoir l'aspect légal. C'est ainsi que nous avons pu démontrer l'utilisation d'un CA dans les entreprises ayant moins de 50 employés, contrairement à nos hypothèses, et dont la fréquence annuelle des réunions démontre qu'il n'est pas simplement accessoire. Il en est de même pour le comité de gestion créé chez les entreprises de plus petite taille.

L'objectif de cette recherche consistait à vérifier la présence d'un conseil d'administration (CA) ou d'un «quasi-CA» (CG) dans la PME manufacturière québécoise. Par un sondage téléphonique effectué auprès de 492 entreprises, dont les informations ont été obtenues pour 362 d'entre elles, nous avons pu montrer que le CA est utilisé formellement dans $29 \%$ des PME, le CG l'est dans $14 \%$ d'entre elles et $57 \%$ des firmes ne recourent à aucun de ces comités.

Cette étude descriptive a permis de mettre en évidence les éléments suivants :

- La taille est un facteur déterminant de la décision de recourir aux services d'un CA ou d'un CG. Les entreprises qui utilisent les services d'un CA sont significativement plus grandes que les autres, alors que celles qui ont créé un $\mathrm{CG}$ sont plus grandes que celles qui n'ont aucun comité.

- L'âge de l'entreprise influence la décision d'utiliser un CA ou un CG. Les entreprises n'ayant aucun comité ont en moyenne moins de 15 ans d'existence.

- Le secteur industriel joue un rôle sur la décision de créer un comité, les entreprises n'ayant aucun comité faisant partie majoritairement du secteur traditionnel.

- Le CA est composé de plus de membres dont un plus grand pourcentage d'externes que le CG des sociétés plus petites.

- Le CA se réunit à une fréquence annuelle, significativement inférieure à celle du CG, dans les entreprises qui font partie des mêmes secteurs industriels.

- Un petit nombre d'entreprises semble confondre le CA, le CG et le comité exécutif. 
Cette première étude empirique de l'utilisation du CA dans la PME n'a permis que de confirmer son existence. Nous savons maintenant que même certaines petites entreprises (moins de 50 employés) utilisent formellement un CA. Nous savons, également, que les craintes de poursuites légales n'ont aucune influence sur leur décision de se doter de cet outil de gestion. Nous ignorons, toutefois, si les caractéristiques individuelles du propriétairedirigeant influencent ce choix, d'une part, et d'autre part si ce choix a des effets positifs sur la performance de l'entreprise.

Plusieurs éléments de l'environnement du CA dans la PME restent à étudier. Notamment, comment les administrateurs sont-ils rémunérés ? Existet-il des liens entre l'environnement de l'entreprise et la composition, la taille et la rémunération du CA ? Qui sont les administrateurs externes qui acceptent d'investir une partie de leur temps dans des comités de petite entreprise ? Quelles sont, ensuite, les conséquences de la présence de tels administrateurs dans la performance de la PME ? Et utilise-t-on de façon aussi formelle le « quasi-CA » par rapport au CA ? Quels rôles jouent ces comités ? Ces rôles sont-ils influencés par la présence d'administrateurs externes ? Ce sont là autant d'avenues de recherche pour une meilleure compréhension des modes de fonctionnement des PME. 


\section{Annexe}

\section{Groupes d'activités composant les trois secteurs industriels*}

(1) Secteur traditionnel : environnement présumément moins complexe Industries des aliments

Industries des boissons

Industries du tabac

Industries du cuir

Industries du textile

Industries du vêtement

Industries du meuble

(2) Secteur de la transformation des ressources

Industries du bois

Industries du papier et des produits en papier

Industries de première transformation de métaux

Industries de la fabrication de produits métalliques

Industries des produits minéraux non métalliques

Industries des produits du pétrole et du charbon

(3) Secteur moderne : environnement présumément plus complexe

Industries du caoutchouc

Industries du plastique

Imprimerie et industries connexes

Industries de la machinerie

Industries du matériel de transport

Industries des produits électriques et électroniques

Industries chimiques

* Nous avons divisé les entreprises en trois grands secteurs industriels selon leur niveau de complexité présumé. Nous avons utilisé en partie les travaux de Julien et Thibodeau (1991) pour effectuer ces regroupements basés sur l'indice de capitalisation et sur l'intensité du travail. 


\section{Bibliographie}

ALDRICH, J.H. et F.D. NELSON (1986), Linear Probability, Logit and Probit Models, Series : Quantitative Applications in the Social Sciences, a Sage University Paper, California, Sage Publications Inc.

BAYSINGER, B. et R.E. HoSKISSON (1990), « The composition of boards of directors and strategic control : Effects on corporate strategy ». Academy of Management Review, vol. 15, n 1, p. 72-87.

BAYSINGER, B., R. KoSNIK et T. TURK (1991), « Effects of board ownership structure on corporate R-D strategy », Academy of Management Journal, vol. 34, $\mathrm{n}^{\circ} 1$, p. 205-214.

BRICKLEY, J.A. et C.M. JAMES (1987), « The takeover market, corporate board composition and ownership structure : the case of banking », The Journal of Law and Economics, vol. 30, p. 161-181.

CASTAldi, R. et M.S. WORTMAN (1984), « Boards of directors in small corporations : an untapped resource », American Journal of Small Business, vol. 9, n 2, p. $1-9$.

ChampouX-Paillé, L. (1992), « Pour des conseils d'administration plus efficaces et plus performants », Mini-conférence de la Chambre de Commerce du Montréal métropolitain, novembre.

Danco, L.A. et D.J. Jonovic (1981), Outside Directors in the Family Owned Business, The Center for Family Business, Cleveland, University Press, 207 p.

FORD, R.H. (1986), Outside Directors and the Privately-Owned Firm : A Study of the 500 inc., Thèse de doctorat, non publiée, Université de Syracuse, 231 p.

FORD, R.H. (1987), « The value of outside directors : myth or reality ? », Business, p. 44-48.

FORD, R.H. (1988), «Outside directors and the privately-owned firm : are they necessary? », Entrepreneurship Theory and Practice, vol. 13, p. 49-57.

FORD, S.J. (1980), "Involving new directors in small company management ", Harvard Business Review, p. 170-174.

Fox, H.W. (1984), «Quasi-boards guidance without governance », American Journal of Small Business, vol. 9, $\mathrm{n}^{\circ} 1$, p. 12-18.

GASSE, Y. et C. CARRIER (1992), Gérer la croissance de son entreprise, Montréal, Éditions de l'entrepreneur, $376 \mathrm{p}$.

JAIN, S.K. (1980), « Look to outsiders to strengthen small business boards ». Harvard Business Review, p. 162-170.

JENSEN, M.C. (1994), « Reviving internal corporate control systems », Financial Management Collection, vol. 9. $\mathrm{n}^{\circ} 1$, p. 12-16. 
JULiEn, P. A. et L. RAYMOND (1991), «Facteurs discriminants de l'adoption des nouvelles technologies dans les PME de service », Entrepreneuriat, PME et politiques gouvernementales, Actes du $8^{\mathrm{e}}$ colloque, CIPE Canada, TroisRivières (Canada), p. 99-112.

Julien, P. A. et J.-C. ThiBodeau (1991), Nouvelles technologies et économie, Les Presses de l'Université du Québec, Québec, Canada.

KORN/FERRY (1990), «Board of directors fourth annual study in Canada », Korn/ Ferry International, $27 \mathrm{p}$.

MaCe, M. (1986), Directors : Myth and Reality, édition revue, Boston, Harvard Business School Press.

Nelson, S. (1985), « Bringing an outside board aboard », Nation Business, p. 30-31.

PEARCE II, J.A. et A.Z. SHAKER (1991), « The relative power of CEO's and board of directors : association with corporate performance », Strategic Management Journal, vol. 12, p.135-153.

RICHARD, J.E. (1989), "Director pay developments in smaller and private companies », Directors \& Boards, vol. 13, n 3, p. 35-39.

Robinson, R.B. (1982), "The importance of 'outsiders' in small firm strategic planning », Academy of Management Journal, vol. 25, n 1, p. 80-93.

Robitaille, L. et J. ST-PIERRE (1993), « Le conseil d'administration dans la PME québécoise : proposition d'un modèle descriptif », Actes du Congrès international francophone de la PME, Carthage (Tunisie).

Roseinstein, J. (1988), «The board and strategy: venture capital and high technology », Journal of Business Venturing, p. 159-170.

Roseinstein, J., A.V. BRuno, W.D. Bygrave et N.T. TAYlor (1993), « The CEO, the venture capitalists, and the board ». Journal of Business Venturing, vol. 8, p. $99-113$.

Roseinstein, S. et J.G. WYATT (1990), « Outside directors, board independance, and shareholder wealth », Journal of Financial Economics, vol. 26, p. 175-191.

Shivdasani, A. (1993), «Board composition, ownership structure, and hostile takeovers », Journal of Accounting and Economics, vol. 16, p. 167-198.

Smeltzer, L. R., B.L. VAn Hook, R.W. Hutt (1991), "Analysis of the use of advisors as information sources in venture startups », Journal of Small Business Management, vol. 29, $\mathrm{n}^{\circ} 3$, p. 10-20.

SPENCERSTUART (1993), Board Trends \& Practices: SpencerStuart Board Index 1993 Proxy Report, Montréal, éd. John S. Hellman, 37 p.

St-Pierre, J., J. M. Gagnon et J. Saint-Pierre (1994), Takeover Bids, Structure of Ownership and Board Composition, Document de travail, Université Laval, Canada. 